\title{
Operación espalda mojada y operación Guatemala: los objetivos de la guerra fría en el racismo interno
}

\author{
Rosalinda Méndez González \\ Universidad Estatal de San Diego
}

\section{Resumen}

Este artículo profundiza mediante un análisis los verdaderos motivos de las operaciones Espalda mojada y Operación Guatemala. Aún cuando se aplica un análisis políticoeconómico a los acontecimientos nacionales e internacionales en torno a la "Operación Espalda Mojada", surge una imagen notoria de la coincidencia de intereses y sucesos entre este acto de racismo masivamente orquestado, y las estrategias estadounidenses de la guerra fría. La victoria real de la "Operación Espalda Mojada" no fue en contra de la amenaza de la "invasión de hordas" de miles de extranjeros ilegales destituidos y despojados de sus derechos políticos; sino que ha de encontrarse en la victoria de la "Operación Guatemala", que fue llevada en contra de la amenaza de un gobierno democrático latinoamericano interesado en asegurar el bienestar de su población a través de la expulsión de la verdadera fuerza que ha perpetuado el desmembramiento de su economía.

Palabras clave: operación espaldas mojadas, operación Guatemala, guerra fría, racismo organizado.

\begin{abstract}
This article analyzes the true motivation for "Operation Wetbacks", and "Operation Guatemala". Even though an economical-political analysis is applied to the national and international events around "Operation Wetbacks", a notorious image of coincidental interests and events between this perfectly orchestrated act of racism, and the American strategies of the cold war. The true victory of "Operation Wetbacks" was not against the menace of "horde's invasion" of thousands of illegal aliens who were deprived and from their political rights. It was in the success of "Operation Guatemala", planned against the menace of a Latin-American democratic government that just worked for the welfare of their population by expelling the true powers that perpetually has tried to disarticulate their economy.
\end{abstract}

Keywords: "Operation Wetbacks", "Operation Guatemala", cold war, organized racism. 


\title{
OPERACION ESPALDA MOJADA Y OPERACION GUATEMALA: LOS OBJETIVOS DE LA GUERRA FRIA EN EL RACISMO INTERNO *
}

\author{
Por \\ Rosalinda Méndez González **
}

Durante el verano de 1954, con fundamento legal en el Acta WalterMcCarran, el gobierno federal de los Estados Unidos llevó a cabo, en toda la nación, una cacería masiva y deportaciones de "extranjeros ilegales", proyecto titulado por el gobierno como "Operación Espalda Mojada". En 1954, las redadas condujeron a las siempre elevada deportación de 1'075,168 personas de las que la mayoría eran extranjeros mexicanos. ${ }^{1}$

Esta operación ha sido tradicionalmente interpretada como una acción de trasfondo racial por parte del gobierno de los Estados Unidos y de los medios de comunicación, y como otro intento más para privar a chicanos y a los inmigrantes mexicanos de sus derechos políticos. ${ }^{2}$

En tanto que ha sido documentada la estrategia de deportaciones y privaciones de los derechos políticos de los inmigrantes y de los mexicoamericanos en la "Operación Espalda Mojada", existen puntos importantes que dicha operación plantea. Primero, dado que el propio gobierno ha propiciado y estimulado la inmigración indocumentada (tan recientemente, como que en enero de 1954 el gobierno se involucró reclutamiento masivo fronterizo y en la "legalización" de extranjeros ilegales, en desafío a la oposición del gobierno mexicano), y que la combinación de fuerza de trabajo bracera e ilegal (esta última constituye la mayor parte de la fuerza de trabajo) ha probado ser muy satisfactoria en el rendimiento de beneficios económicos, en el mantenimiento de una reserva de fuerza de trabajo para la agricultura, y en la prevención de los

\footnotetext{
- Traducido por: Guadalupe Ortega Villa.

Este artículo apareció publicado en la revista "Research in Political Economy" Vol. 10. Editado por Paul Zarembka. JAI Press Inc.

** Profesora asociada del Departamento de Estudios Mexicoamericanos de la Universidad Estatal de San Diego. San Diego, Ca., EUA.

1 Este estudio fue llevado a cabo en 1980-1981 como parte de tesis doctoral de la autora (González, 1981). Parte de este análisis fue presentado con una ponencia en 1982 en Washington, D.C. en el Encuentro de la Asociación de Estudios Latinoamericanos, bajo el nombre de "The Post-War Politics of U.S.-Latin America Inmigration Policy".

${ }^{2}$ La "Operación Espalda Mojada" está discutida, entre otros, en: Acuña, 1981; Grebler, 1965; Guzmán, 1976; Morgan, 1954; Reavis, 1978; Samora, 1971; García, 1980. El único documento publicado en el cual se ha tratado comprensiva y ampliamente el estudio de la Operación Espalda Mojada, es en el libro de Juan Ramón García, Operation Wetback: The Mass Deportation of Mexican Undocumentated Workers in 1954 (1980). García, sin embargo, no ofrece la explicación presentada en este documento, pero centra su interpretación en los males que afronta Estados Unidos internamente, y considera a la "Operación Espalda Mojada" como un intento para distraer la atención pública de esos males. El análisis que aquí se presenta, desde mi punto de vista, no ha sido realizado anteriormente.
} 
esfuerzos del sindicalismo agrícola, ¿por qué tan repentinamente y de manera tan radical, el gobierno cambió su estrategia?. Segundo, ¿́por qué la operación fue llevada a cabo de tal manera - esto es, con una publicidad masiva, previa, anunciando el lugar y el tiempo tentativo para la redada, eliminando así el importante elemento sorpresa tan esencial para el éxito de las redadas normales? ¿por qué los agricultores apoyaron, tan efusivamente, la operación cuando en el pasado ellos se habían opuesto constantemente a todos los esfuerzos legislativos y burocráticos para terminar con la inmigración ilegal? ¿Por qué el presidente Eisenhower y el procurador general Brownell -al principio muy desinteresados en el problema de los extranjeros ilegales - cambiaron sus actitudes tan repentinamente de la noche a la mañana? y, ¿por qué tan súbitamente enfriaron el asunto después de julio de 1954? ¿Por qué fue llevada a cabo la operación con tal cooperación masiva de la federación, de los militares, del gobierno local, y de la prensa? y ¿por qué fue llevada a cabo precisamente en ese entonces?

La duración de la "Operación Espalda Mojada" coincidió notablemente con el golpe patrocinado por la CIA, "Operación Guatemala", el cual echó abajo al presidente de aquella nación centroamericana que había sido electo democráticamente. Además, los sucesos y personajes en torno a la "Operación Guatemala" tienen cierta complementariedad con aquéllos en torno a la "Operación Espalda Mojada".

Cuando el presidente Eisenhower tomó posesión, en enero de 1953, él y su secretario de Estado, en un principio, no prestaron atención a Latinoamérica (Gerassi, 1963, p. 222). Ni Eisenhower ni su procurador general estaban interesados en el asunto de la inmigración ilegal. De hecho, el procurador general señaló que "él tenía pensado hacer poco acerca de esa situación y que por el momento el asunto de los 'espaldas mojadas' no era prioritario para él”. Además, "dijo al Comité de los Bienes de la Casa que él se oponía a otorgar un incremento de fondos a la Patrulla Fronteriza, ya que en su opinión simplemente no era necesaria". (Garé́a, 1980, p. 157).

Pero repentinamente, en 1953, el presidente de Guatemala, Jacobo Arbenz Guzmán, nacionalizó los bienes de la United Fruit Company en su país, con un valor de $\mathbf{4 0}$ millones de dólares, como parte del programa masivo de reforma agraria. Eisenhower y su secretario de Estado repentinamente se interesaron mucho en América Latina. Para finales de 1953 Eisenhower determinó que Arbenz tenía que irse. Se dirigió al director de la CIA, Allen Dulles, y el diseño de la "Operación Guatemala" se puso en marcha. (Wise y Ross, 1964, p. 171.).

\section{1. "OPERACION ESPALDA MOJADA" Y "OPERACION GUATEMALA"}

Cuando Eisenhower llegó a la presidencia en 1953, entre los hombres que designó para formar parte de su gabinete, se encontraban 
John Foster Dulles como secretario de Estado, y su hermano Allen Dulles como director de la CIA. Los hermanos Dulles y algunos otros designados por Eisenhower, estaban profundamente involucrados con la United Fruit Company (entre otras corporaciones), cuyas inversiones incluían cerca de 2'000,000 de acres en plantaciones de plátano en América Central, Ecuador y Colombia; miles de acres de caña de azúcar, cacao y palma aceitera, 1,500 millas de ferrocarriles, 60 barcos, refinerías de azúcar, puertos, y redes de comunicación en Centro y Sudamérica (Gerassi, 1963, p. 151). El pulpo de la United Fruit tenía relaciones estrechas con el First National Bank de Boston y con la administración de Eisenhower. Algunas de las conexiones revelaron: que a) el despacho jurídico de Foster Dulles había escrito los planes de los acuerdos de 1930 y 1936 de la United Fruit Company con Guatemala; $b$ ) el director Allen Dulles había sido presidente de la United Fruit; c) John Moors Cabot (en ese entonces secretario de Estado Asistente para Asuntos Interamericanos, y recientemente embajador de los Estados Unidos en Brasil) y su familia fueron accionistas de la United Fruit (Gerassi, 1963, p. 153).

Uno de los representantes subsidiarios de la United Fruit Company, ya en 1920 había puesto en claro los objetivos de la compañía para América Central en una carta que contenía una serie de instrucciones, dentro de las cuales estaba,

6. Estos hombres (los centroamericanos y sus gobiernos) no deben de actuar por su propia iniciativa; deben de hacerlo de acuerdo a determinadas condiciones, y bajo nuestro control directo.

...

9. Debemos desmembrar la incipiente economía de este país a fin de incrementar y ayudar a nuestros propósitos. Debemos de prolongar su trágica, tormentosa y revolucionaria vida; el viento sólo debe de soplar en nuestros barcos y el agua sólo debe mojar nuestra quilla (subrayado nues. tro) (Gerassi, pp. 215-216).

Cuando Allen Dulles recibió, en 1953, instrucciones acerca de Arbenz, la CIA elaboró un plan muy sofisticado, que no sería la primera intervención de la CIA en gobiernos extranjeros, ni su primer esfuerzo para derrocar un gobierno democráticamente electo, De hecho, en ese año anteriormente se había llevado a cabo exitosamente un "golpe" en Irán, cuando Eisenhower decidió que el Primer Ministro nacionalista Mohammed Mossadegh "se debía de ir". La CIA lo sustituyó por el ahora impopular Sha Mohammed Reza Pahlevi(Barnet 1972, pp. 202, 203, Wise y Ross 1964, pp. 110-114). Sin embargo, un golpe guatemalteco era una operación especialmente delicada, dada la sensibilidad de Latinoamérica a los golpes de estado y la desconfianza hacia los Estados Unidos. Este llegó en un período de deterioro de las relaciones Estados UnidosLatinoamérica, al mismo tiempo que los Estados Unidos pugnaban por una agresiva política anticomunista en América Latina, y presionaban a 
1a Organización de Estados Americanos para que adoptara tal política. Estados Unidos no era el único que observaba de cerca la situación guatemalteca y sus reformas populares. El cambio en Guatemala también era observado favorablemente por las naciones latinoamericanas.

Por lo tanto, era esencial para los planes de intervención de la CIA en Guatemala, que se mantuviera absoluta discreción al respecto. Y es precisamente en esta coyuntura que Eisenhower se interesó en el asunto de los "espalda mojada", ordenándole al procurador general Brownell que investigara y elaborara un plan. En agosto de 1953 Brownell voló a California, aproximadamente al mismo tiempo en que Eisenhower envió al seleccionado de la CIA, John Emil Peurifoy, para que se uniera a las operaciones de la CIA como embajador en Guatemala (Ross y Wise, 1964, pp. 170-171). La misión de Peurifoy fue llevada a cabo con el mayor de los secretos y la misión de Brownell "uno de los problemas más importantes en espera de (su) resolución", se realizó con el máximo de publicidad en todos sentidos (García 1980, p. 171).

En enero de 1954, Estados Unidos logró que la OEA (Organización de Estados Americanos) aprobara la llamada "Declaración de Caracas", pasando por alto el principio de no intervención en aras del "anticomunismo" (Aguilar Monteverde 1955: 134-137). En marzo de 1954, Brownell sostuvo una conferencia en la que prometió que actuaría de inmediato para abordar el asunto de los "espaldas mojadas". Sin embargo, Brownell no hizo nada durante varios meses sino hasta el 9 de junio, cuando anunció los planes de una redada masiva de extranjeros indocumentados y del envío de una propuesta al Congreso, para frenar la migración ilegal (García, pp. 159-160).

Todo el complicado plan de la "Operación Espalda Mojada" fue llevado a cabo con precisión militar. Como parte de la campaña, y por primera vez, un militar fue nombrado para hacerse cargo del Servicio de Inmigración y Naturalización: el general Joseph May Swing. Este había ocupado formalmente el cargo de comandante de la Sexta Armada en California, retirándose en 1954 con el rango de teniente general de la Armada de los Estados Unidos. Compañero de clase de Dwight Eisenhower en West Point, y trabajó con el general John Pershing en 1916 en la "expedición punitiva" en contra de Pancho Villa. "Swing tenía fama de ser "alguien que odiaba a los mexicanos desde hace mucho tiempo, y de manera profesional'..." (García, 1980, p. 172).

Swing, solicitó intervenir el 24 de febrero de 1954, dándole a la operación "las tácticas y la retórica de una campaña militar" - pero de una campaña militar de la que cualquier movimiento y cualquier táctica eran deliberada y ampliamente dados a conocer con anticipación (Reavis, 1978, p. 43). La estrecha amistad de Swing con Eisenhower fue tan importante para la operación como su posición militar. Durante el proceso de campaña, tuvo varias entrevistas con Eisenhower "sin contar con una cita previa" 
(García 1980:172). La atención de la nación, y sin duda la de América Latina (ya que incluso el Servicio Informativo de los Estados Unidos estaba involuerado en la operación), fue atrapada por ésta altamente coordinada guerra relámpago de "seguridad nacional", la cual tenía la aprobación de los conductos más altos de Washington. Las especificidades de la estrategia fueron decididas en el Departamento de Estado en mayo de 1954. (García, p. 175) Entonces...

Asistida por autoridades federales, estatales, del condado y municipales -incluyendo oficiales policiacos del ferrocarril, oficiales de aduana, el FBI, la Armada y la Fuerza Naval-y apoyados por aeronaves, barcos, automóviles unidades de radio, fuerzas especiales, y quizá lo más importante de todo, el sentimiento del público, incluyendo el de los agricultores; la Patrulla Fronteriza emprendió la ofensiva más grande en tiempos de paz, en contra de la fuertemente explotada, desorganizada y carente de estructura "fuerza invasora" de migrantes mexicanos (Samora, 1971, p. 52).

La "Operación Espalda Mojada" (como la llamó el procurador general) dio inicio el 17 de junio de 1954, con la aprobación del presidente Eisenhower y con la cobertura de la prensa radio y televisión. La "Operación Guatemala" (como la Ilamó la CIA) principió el 18 de junio de 1954. Ross y Wise describen los sucesos:

El golpe de la CIA en contra del régimen dominado por el comunismo de Jacobo Arbenz Guzmán había empezado. Esa tarde el coronel Carlos Castillo-Armas, un exiliado guatemalteco entrenado por los Estados Unidos, cruzó la frontera de Honduras con aproximadamente 150 hombres. La invasión estaba en marcha. Tenía el consentimiento previo del presidente Eisenhower. . .

[pero lo que] la CIA tenía planeado como un golpe de un día se demoró doce difíciles días. Antes de que terminara, Peurifoy estaba profundamente involucrado en una operación política encubierta. El presidente de los Estados Unidos. . . vio la necesidad de enviar clandestinamente tres aviones de guerra más y aminorar la revuelta infructuosa de la CIA. . Muchos meses de cuidadosa preparación habían sido dedicados a la invasión de Castillo-Armas (Ross y Wise, 1964, pp. 165-166).

Cuando la "Operación Guatemala" terminó, John Foster Dulles la ealificó como una "victoria gloriosa" (Aguilar Monteverde, 1965, p. 138). Brownell igualmente feliz, calificó su sincronizada "Operación Espalda Mojada" como un "tremendo éxito" (García 1980, p. 200). El secreto fue crucial para el éxito del primero, y crucial para el último, fue la publicidad. El análisis exhaustivo de Ramón García sobre la "Operación Espalda Mojada" señala:

Desde el principio, la cobertura de los medios de comunicación había sido un factor importante en el conjunto de los planes de la operación... para eliminar todo elemento sorpresivo... al proyecto se le dio amplia publicidad. .. mucha de la... 'propaganda' fue el resultado de los 
esfuerzos específicos y producto de la información del Servicio de Inmigración, que vio que esa cobertura era esencial para sus planes. . . Como H.L. Landon le reportó a Swing, 'la cobertura deseada' ha sido arreglada 'por lo tanto esa muy favorable publicidad' será obtenida, "no sólo a nivel local sino nacional . . . La rápida intervención cuidadosamente planeada de los medios de comunicación logró sus propósitos. (subrayado nuestro) García, 164).

Un objetivo que no se cumplió fue la reforma legislativa prometida. Para fines de agosto, tan pronto como terminó su "tremendamente exitoso" ataque a través de los medios de comunicación (y aun antes de que terminara su campaña militar), Brownell -y la administración de Eisenhower- dejaron caer el asunto de los "espalda mojada" como una papa caliente. La autorización de la iniciativa de ley prometida estaba siendo debilitada, y

La repentina falta de iniciativa mostrada por Brownell para luchar por la iniciativa de ley ante el Congreso fue lo que molestó a aquéllos que apoyaban su autorización. Para fines de agosto el procurador general era atacado por la fuerza de los representantes de la fuerza de trabajo, y por los grupos religiosos; por permitir que su tan anunciado programa legislativo tuviera tropiezos en el Congreso. . . De hecho, el Comité Judicial del Senado no recibió los borradores de la iniciativa de ley sino hasta tres meses después de que a Brownell se le comisionara el asunto de la legislación. . . En lo que [al senador Morse] concernía, la responsabilidad de la no autorización de la propuesta de ley descansaba totalmente sobre los hombros de Eisenhower y su administración (subrayado nuestro) (García, 164).

Tan pronto como Eisenhower y su administración habían pasado del frío al calor en el asunto los "espalda mojada", ahora se iban del calor al frío. El otro objetivo fue el logro de la campaña ampliamente publicitada. García intuye que “'La Operación Espalda Mojada' y la propaganda que le acompañó sirvieron una vez más para desviar la atención de los verdaderos problemas en México y Estados Unidos, que contribuyeron a la entrada de 'ilegales' " (p. 232). Fundamentalmente, en mi opinión, sirvió para desviar la atención de la nación lejos de una más peligrosa, inalcanzable y "exitosamente brillante" intervención de los Estados Unidos en el exterior.

\section{EL SIGNIFICADO DE LAS " OPERACIONES"}

¿Cuáles son las lecciones históricas de estos sucesos? ¿Cuál era el contexto en el que ocurrieron? y ¿cómo difiere del contexto mundial de hoy? Para comprender esto, sería de ayuda observar cómo los eventos posteriores a la Segunda Guerra Mundial influenciaron los sucesos internos y de la Unión Americana de la década de los cincuenta. 


\section{A. Los Estados Unidos y la situación internacional ${ }^{3}$}

La Segunda Guerra Mundial alteró, en la mayoría de los casos, la situación nacional e internacional. Internacionalmente, los Estados Unidos surgieron como la fuerza suprema del mundo. Su penetración económica, política y militar en la Europa Occidental y en el Tercer Mundo fue fuertemente extendida en virtud de su capacidad para proporeionar crédito, armamento, y productos industriales a los países beligerantes; su localización geográfica le permitió participar en la guerra sin sufrir ataques o invasiones en su territorio continental, y sin la desvastación física y económica de los países europeos y asiáticos después de la guerra.

Este dominio internacional fue retado por dos grandes amenazas que enfrentó Estados Unidos en el período de la posguerra. Uno fue el surgimiento de los movimientos nacionales de liberación e independencia estimulados por la propia guerra. Polonia, Checoslovaquia, Yugoslavia, Rumanía, Hungría, Bulgaria y Albania adoptaron el socialismo. La República Democrática Alemana nació de la guerra. China, con una cuarta parte de la población mundial, logró su revolución y estableció una república popular. En Corea y en Vietnam se establecieron repúblicas populares. En conjunto, estos países representan más de la tercera parte de la población del mundo.

En otros países, la independencia de la dominación colonial directa también fue lograda. India, Indonesia, Birmania y Ceilán adquirieron y buscaron mantener su independencia. Las luchas armadas por la liberación brotaron en Malasia, Filipinas, Madagascar, Costa de Oro en Africa, Kenia, Sudáfrica, Persia, Egipto, Argelia, Túnez, Marruecos. En Latinoamérica, surgieron movimientos populistas antiimperialistas en muchos países.

La segunda gran amezana a la que se enfrentó Estados Unidos estaba relacionada con estos acontecimientos: el fortalecimiento de la URSS y el surgimiento del territorio socialista, particularmente con el triunfo de la revolución China y la intervención soviética en Europa Oriental.

Estados Unidos respondió a estas amenazas a través de un proceso doble, interno (examinado más adelante en la sección "B"),e internacional. Internacionalmente, buscó consolidar su influencia a través de programas de "ayuda externa" y de la creación de agencias internacionales de planeación gubernamental y de cuerpos y alianzas militares; de este modo se expandió profundamente el capitalismo monopolista de estado en todo el mundo no socialista (en ambos, en el Tercer Mundo y en Europa Occidental y Japón).

La institucionalización e internacionalización de estos ataques fue efectuada por el Consejo de Seguridad de las Naciones Unidas, de la

\footnotetext{
${ }^{3}$ Las fuentes de este apartado incluyen a: Blum, 1968; Staley, 1961; Stoessinger, 1961; Freymond, 1964; Divine, 1979; Horowitz, 1969; Barnet, 1972; Hudson, 1972; Josephson, 1972; Aguilar Monteverde, 1965; Gerassi, 1963; Kinder y Hilgemann, 1978.
} 
Agencia de Rehabilitación y Ayuda de los Estados Unidos, y a través de las instituciones de Bretton Woods, del Banco Mundial (Banco Internacional de Reconstrucción y Fomento), el FMI (Fondo Monetario Internacional) y el GATT (Acuerdo General de Aranceles y Comercio). En Europa, la "ayuda" del gobierno de Estados Unidos y los planes de la posguerra fueron efectuados por el Plan Marshall; en América Latina a través del sistema interamericano.

Simultáneamente, Estados Unidos creó una red de sistemas de "alianza" militar; con Europa del noroeste, ta OTAN (Organización del Tratado del Atlántico Norte); con latinoamérica, la OEA (Organización de Estados Americanos); con Australia y Nueva Zelanda, el Pacto ANZUS (Australia, Nueva Zelanda y los Estados Unidos); para la "protección" de los estados asiáticos del Sureste, la SEATO (Organización del Tratado del Sureste de Asia, cuyos únicos miembros del Sureste asiático son Filipinas y Tailandia); con Turquía, Irán, Pakistán y Bretaña, el CENTO (Organización del Tratado Central), y otros pactos bilaterales en el Pacífico.

El uso de "ayuda" económica como un medio de dominación no era nuevo. Ya a principios de 1933, cuando el Presidente Roosevelt proclamó la política de los "buenos vecinos" para América Latina, diseñada para buscar una mayor "cooperación interamericana" y abandonar la "diplomacia del dólar", este cambio de tácticas fue acompañado por la creación (en 1934-35) del Banco de Exportaciones e Importaciones para proporcionar crédito a América Latina. El objetivo de lograr la dominación apoyándose en medios económicos más que en militares, requería, como Roosevelt reconoció, de que:

Los intereses del gobierno y de la iniciativa privada. . debían de ser invertidos en América Latina a fin de 'desarrollar recursos de materias primas requeridas en los Estados Unidos'. El 26 de septiembre de 1940 [Roosevelt] incrementó los límites de crédito del Banco de Importaciones y Exportaciones de $\$ 100$ millones a $\$ 700$ millones de dólares, y para el día del ataque a Pearl Harbor, la mayoría de las naciones latinoamericanas ya habían recibido préstamos para su "desarrollo". Sin embargo, ni los préstamos ni las inversiones fueron dirigidos para ayudar a aliviar los males económicos de las naciones latinoamericanas, los cuales principalmente eran causados por basar su economía en el monocultivo o en la producción de mercancías. El objetivo de los préstamos, dijo Roosevelt, era forzar a esas naciones para llegar a ser completamente dependientes, en términos económicos, de los Estados Unidos (Gerassi, 1963, p. 220).

De hecho, fue en América Latina el primer lugar en donde los precursores del desarrollo del Programa "Point IV" de Truman, lo Ilevaron a cabo por primera vez bajo la dirección de Nelson Rockefeller y la Oficina de Asuntos Interamericanos que él dirigía. El Programa "Point IV" de Truman introdujo la táctica de dominación a través de la 
"ayuda tecnológica". Mientras que los planes internacionales de Estados Unidos y la exportación de capitales que respaldaban el Plan Marshall tuvieron éxito en el apuntalamiento de la economía europea para impedir el desarrollo revolucionario en esos países, y los proveyó de infraestructura y medios financieros para pagar su deuda con Estados Unidos y "abrir" sus mercados al capital y bienes norteamericanos, los objetivos del Programa "Point IV" fueron frenar las corrientes revolucionarias en el Tercer Mundo mientras que transformaban sus economías en mercados para los excedentes de producción industrial estadounidense. Truman describió el Programa "Point IV" como:

"un atrevido programa nuevo para hacer que los beneficios de nuestro adelanto científico y nuestro progreso industrial serán accesibles para el mejoramiento y crecimiento de las regiones subdesarrolladas". Con la Europa Occidental supuestamente segura ante el comunismo, la siguiente prioridad era prevenir que la restante tercera parte del mundo cayera en manos comunistas. (Blum, 1968, pp. 776-777).

Estados Unidos enfrentó el reto de la guerra fría soviética y las luchas acaloradas del Tercer Mundo, a través de un programa masivo de militarización de la economía y del gobierno. Este incluyó la racionalización y el fortalecimiento de los sistemas existentes, la expansión y acumulación de las reservas de armamento nuclear, la repentina apertura del programa de "investigación espacial", el establecimiento de bases militares en todo el mundo; institucionalizando el reclutamiento, y creando agencias especiales de espionaje internacional y de fuerzas contrarrevolucionarias.

Un aspecto importante de estos intentos coordinados por los Estados Unidos para imponer la estabilidad política y "abrir la puerta" (regulada por los Estados Unidos) para el flujo mundial de tropas, bienes, servicios, préstamos y pagos, fue la regulación del trabajo y de la migración. En las Naciones Unidas, mientras los flujos de la agricultura y otras mercancías eran supervisados a través de agencias tales como la FAO (Organización de la Agricultura y Alimentación) y la Organización Internacional del Comercio; la mercancía fuerza de trabajo fue asignada a una agencia especial, la Organización Internacional del Trabajo.

El establecimiento del sistema mundial de "puerta abierta" que Estados Unidos buscó imponer, implicó una división internacional del trabajo, dentro de la cual los altos precios del capital y de los artículos estadounidenses fluirían a las puertas abiertas de los países capitalistas y del Tercer Mundo, mientras que las puertas abiertas de los países del Tercer Mundo permitían la salida de sus materias primas y fuerza de trabajo baratas hacia los Estados Unidos y otros países capitalistas. Pero en cualquier caso, Estados Unidos retenía los controles sobre las entradas y las salidas de estos flujos, mientras el resto de la comunidad del "mundo libre" estaba forzada a mantener sus puertas entreabiertas y sin ningún control. 


\section{B. La guerra fría interna ${ }^{4}$}

Así como la Segunda Guerra Mundial le permitió a Estados Unidos extender su dominación a nivel internacional, internamente le representó las condiciones para consolidar la dominación del estado y establecer el capitalismo corporativo. Un aspecto de esta consolidación fue el tremendo crecimiento y el surgimiento en importancia de la milicia en la economía y en el gobierno. La guerra proporcionó los motivos para el abandono de las políticas del "New Deal" y para transferir el poder hacia los militares y partidarios de la maquinaria bélica: los grandes industriales y los banqueros fueron quienes se beneficiaron de estas alianzas con los militares. La creación de la Unión de Jefes del Estado Mayor, que incrementó significativamente el papel de los militares en el gobierno, fue un indicador de la manera antidemocrática en que estas transferencias de poder fueron llevadas a cabo. Roosevelt pasó por alto al Congreso y simplemente creó y trajo esta centralización de la unidad militar al gabinete del gobierno federal, con profundas implicaciones para el futuro de las políticas americanas tanto internas como externas. Esta intervención de los militares dentro de los dirigentes nacionales, fue sostenida y expandida después de la guerra:

El desarrollo de las fuerzas de Estados Unidos en todo el mundo al finalizar la guerra, representó una oportunidad para proyectar el poder de una nación que no quería renunciar a hacerse cargo de las 'responsabilidades como dirigente mundo'. Así, la Junta de Jefes del Estado Mayor discutió exitosamente la retención de la mayoría de la red de bases adquiridas durante la guerra. El programa de investigación y desarrollo, las relaciones públicas y las redes de propaganda; los programas de asistencia militar, y los aparatos de inteligencia y subversión, los cuales difícilmente existían en 1940, permanecieron para ser los principales receptores de los fondos del gobierno después de que volvió la paz (Barnet, 1972, p, 30).

La creación de la OTAN, SEATO, la OEA y de otras alianzas militares; el mantenimiento del reclutamiento, la autorización en 1947 de la Acta de Seguridad Nacional, creando un sistema de inteligencia masivo (Agencia Central de Inteligencia, Consejo Nacional de Seguridad, Agencia de Inteligencia de la Defensa, Agencia Nacional de Seguridad, Inteligencia de la Armada, Inteligencia de la Marina, Inteligencia de la Fuerza Aerea, el Buró de Inteligencia e Investigación del Departamento de Estado, la Comisión de Energía Atómica, Buró Federal de Investigaciones; y otros [Wise y Ross, 1964] y la proliferación de guerras, revoluciones,

\footnotetext{
- Las fuentes consultadas para este apartado incluyen a: Blum, 1968; Divine, 1979; Josephson, 1972; Barnet, 1972; Lens, 1974; McWilliams, 1968, 1964, 1979; Bennett Jr., 1976; Dobb, 1962; Greenleaf, 1970; National Advisory Commission on Civil Disorders, 1968; Green, 1976; Morales, 1972; Endore, 1944, 1948; Citizen's Committee for the Defense of Mexican-American Youth, 1942; Gonzalez, 1947; Guzman 1976; Morgan, 1954; Galarza, 1978; Cochran, 1972; Wise y Ross, 1964.
} 
y "manchas problemáticas", aseguraron la continuación de una economía militarizada y la preminencia del papel de los militares.

El segundo aspecto fue el abrumador alcance de la fusión del gobierno (incluyendo los militares) con los "grandes negocios"; el sólo hecho de la fusión eliminó a los competidores pequeños. Con la centralización extrema durante la guerra y la unión del gobierno, los militares y los sectores financiero/industriales; la banca estadounidense y las corporaciones eliminaron a los pequeños competidores y amasaron miles de millones de dólares en ganancias. En 1938, las utilidades de este monopolio habían sido de 3,300 millones; para 1940, se había incrementado a 17,000 millones; en 1942 a 20,900 millones; y para $1944, \$ 23,300$ millones (Instituto de Economía Política, 1957, pp. 352-353). Con la justificación de la emergencia de guerra, Roosevelt creó agencia tras agencia para reemplazar los cuerpos gubernamentales normales. Ubicó a los grandes banqueros y a los industriales al frente de estas agencias, dándoles poder absoluto para planear y poner en movimiento la economía, la vida politica, y hasta los canales de propaganda y manipulación ideológica. Entre estos: Eduard R. Stettinius, presidente de Aceros de Estados Unidos, fue ubicado como la cabeza de la Junta de Recursos de Guerra; "el Gran Bill" Knudsen, presidente de la General Motors y admirador de Hitler, estuvo a cargo de la producción de armamentos; Ralph Davies, vicepresidente de la Standard Oil de California, como coordinador de la Administración de Petróleo, Donald Nelson, vicepresidente de la Sears Roebuck, encabeź́ la Junta de la Producción de Guerra, etcétera (Josephson, 1972; Cochran 1972).

Esta fusión del capital industrial y financiero con el gobierno y li milicia, creó el capitalismo monopolista de estado bajo la forma de "economía de defensa", dentro de la cual el gobierno se convirtió en e. primer suscriptor, así como en el principal cliente de las corporacionet más importantes. Las 200 corporaciones más grandes recibieron alrede. dor del 70 por ciento de los contratos masivos de la guerra, mismos que continuaron creciendo a medida que el insaciable apetito militar iba más allá de los requerimientos de guerra. De este modo, las principales preocupaciones industriales "pasaron a estar felizmente casados con el enorme aparato militar del gobierno", según palabras de Matthew Josephson. (1972, p. 262).

Un tercer aspecto de esta dominación fue el rumbo hacia el fascismo dentro de la vida política de los Estados Unidos. La década de 1940 presenció el incremento de los ataques en los grupos de ciudadanos residentes tales como los japoneses, chicanos y negros, como parte de un ataque nacional más amplio hacia la fuerza de trabajo y a las organizaciones políticas de izquierda.

La autorización del Acta Smith en 1940, violando los derechos constitucionales de libertad de expresión y libertad de pensamiento, y 
haciendo de la enseñanza o de la apología de la revolución un acto criminal, justificó la persecución del Partido Comunista y de sus simpatizantes. Esto fue seguido en 1942 por el Acta Smith Connally. En junio de 1947, el Acta Taft-Hartley fue autorizada, reprimiendo el derecho a huelga de los trabajadores, estropeando la difusión del sindicalismo hacia industrias y regiones desorganizadas en donde las minorías étnicas estaban concentradas, $y$ forzando a hacer una purga de los trabajadores comunistas y militantes de los sindicatos de trabajadores.

Un elemento importante del "problema de la fuerza de trabajo" fueron las minorías segregadas. La rápida urbanización durante la guerra y la maduración de la segunda y tercera generación de los hijos de las minorías étnicas, quienes eran menos dóciles y estaban conscientes de sus derechos como ciudadanos, generaron una creciente e incesante militancia en las ciudades. Carey McWilliams relata las experiencias de los chicanos (ciudadanos de ascendencia mexicana nacidos en los Estados Unidos) de las áreas rurales del suroeste quienes fueron reclutados en el ejército y tuvieron que ser asignados a una unidad especial iporque no hablaban inglés! La guerra transformó sus experiencias, como lo hizo con muchos otros chicanos:

En cada fase de la guerra, incluyendo los planteles de defensa y las escuelas de entrenamiento así como los servicios armados, se abrieron oportunidades semejantes para miles de mexicoamericanos: para aprender nuevos oficios, adquirir nuevas experiencias; para entrar en contacto con corrientes completamente nuevas de pensamiento y opinión. En más de una comunidad, la incorporación de civiles a varias agencias de servicios de defensa, tuvo una marcada tendencia a romper las barreras que durante tanto tiempo separaron a los hispanoamericanos del resto de la población. Esta experiencia de guerra, como se podía esperar, trajo un nuevo orgullo en el hecho de ser ciudadano y un resentimiento creciente en todas las formas de discriminación (MeWilliams, 1968, p. 260).

A pesar de las tácticas de segregación étnica y discriminación racial, la inclusión de la población chicana dentro del proletariado y de las tendencias inherentes de producción industrial moderna para romper con las diferencias entre la gente, ha contribuido a su integración y a su despertar político.

Los acontecimientos en California presentaron un microcosmos de esta situación y una cristalización de la respuesta a esta amenaza. California fue un pionero en la importación de peones chinos y del Tercer Mundo. En parte, esto se debió a su situación geográfica.

Localizada en el límite oeste del continente y en la orilla este del Pacífico, la agricultura de California está estratégicamente ubicada con respecto de las reservas de fuerza humana de China, Japón, Filipinas, el valle del Misisipi y de México (Galarza, 1978, p. 34). 
Como resultado, las principales minorías étnicas estuvieron fuertemente representadas en California. Los Angeles concentró a los tres grupos principales: mexicanos, asiáticos y negros. El potencial político que esta concentración presentaba era claro. En 1947 McWilliams señaló que:

Con una población mixta de aproximadamente 500,000 , negros y mexicanos tendrán una profunda influencia en el futuro de Los Angeles. , , Una vez que empiecen a colaborar en asuntos políticos, sociales y económicos, constituirán un significativo elemento de equilibrio de poder en la población. (McWilliams, 1975, pp. 325-326).

La guerra proporcionó la at mósfera adecuada para la toma de acción política para impedir este potencial.

Las demandas de los trabajadores de la producción bélica y su movilización, han llevado a la urbanización de las familias negras, al igual que las chicanas a su mayor incorporación en la industria y en las fuerzas armadas. En respuesta a la continua discriminación y a la mala situación económica, la comunidad negra empezó a movilizarse en toda la nación. Se desarrolló un movimiento mediante una "marcha a Washington", con reuniones y actividades en todo el país para coincidir masivamente en la capital en 1941. Roosevelt, quien se resistió a autorizar las propuestas de ley de derechos civiles, fue forzado a emitir un Decreto (\# 8802) para invalidar la marcha. La medida dejaba fuera de ley la discriminación en el gobierno y en las industrias de la defensa. Mientras este acto de áltimo minuto resultó eficaz en la suspensión de la marcha a Washington; el movimiento sin embargo, tuvo éxito al estimular la acción masiva en los negros.

En 1942, el Congreso de Igualdad Racial fue establecido a fin de diseñar las tácticas de las huelgas sentadas en la década de 1930 y el acceso a actos masivos no violentos como el de Gandhi. La reacción ante este despertar político no tardó en efectuarse. Se iniciaron los ataques raciales en Mobile, Los Angeles, Beaumont (Texas) y en otras ciudades. El 20 de junio de 1943, en Detroit se presentó un disturbio racial, entre los blancos que buscaban su supremacía, y los negros; culminando con la muerte de 25 negros y de 9 blancos. En ese mismo año un disturbio en Harlem, Nueva York, dejó un total de 6 muertos, más de 500 heridos, y arriba de 100 encarcelados (National Advisory Commission, 1968, p. 104).

Esta experiencia también fue sufrida por las comunidades chicanas. Carey McWilliams describe el problema y la solución ad hoc que se le dio:

No importa qué tan grande sea la disparidad del poder social, no es una tarea fácil mantener reprimida a una considerable población minoritaria, en este caso una minoría de aproximadamente 350,000 personas. . . De vez en cuando se le debe "dar una lección" a las minorías, para hacerles enten- 


\section{OPERACION ESPALDA MOJADA Y OPERACION GUATEMALA}

der su papel dentro de un esquema más amplio de cosas. . .El método nuevo que sustituyó los linehamientos, involucra lo que se conoce como una "política de brutalidad", asesinatos, golpizas, y otros actos de violencia protegidos por los censores de la comunidad, con la condena del dogma de que los oficiales de la policía no pueden actuar equivocadamente, y que "el brazo de la ley" debe de ser sostenido a cualquier precio. (Endore, 1948, pp. 3-4).

Tres años después de la autorización del Acta Smith, dos años después del Decreto Ejecutivo 9906, que ordenaba la concentración en campos de los americanos de ascendencia japonesa, y después del establecimiento del "Programa de Abastecimiento de Fuerza de Trabajo Mexicana" que institucionalizaba la importación de la fuerza de trabajo mexicana, y simultáneamente a los disturbios raciales en contra de los negros en las ciudades de todo el país; surgieron los disturbios Zoot Suit entre policías navales y chicanos (y negros) en los barrios del este de Los Angeles y en Watts. Estos grandes ejemplos de la brutalidad oficialmente sancionada, ejercida por las fuerzas armadas en contra de los chicanos se repitieron en muchos lugares de todo el país. (McWilliams, 1968; Morgan, 1954; Citizens' Committee, 1942; González 1947; Guzmán, 1976). Como años antes señaló Max Handman al respecto de la discriminación en contra de los primeros inmigrantes mexicanos, "Un grupo que es traído o atraído con propósitos de explotación económica, llevará consigo el estigma de esa explotación. No es deseado para ningún otro propósito, y pronto se dará cuenta de que no hay lugar para él bajo ninguna otra situación" (Handman, 1926, p. 40).

Las raíces de estos ataques a la fuerza de trabajo y a las comunidades minoritarias parten del período de las intensas luchas de clases que precedieron a la guerra. Las "grandes huelgas" sangrientas de principios de la década de 1930, la militancia en las "huelgas de los sentados" de finales de esa década, y el enorme crecimiento del Partido Comunista durante la gran depresión, hicieron pensar a los grandes patrones que era necesaria una estrategia de doble alcance en contra de los trabajadores. Reconociendo que los sindicatos estaban creados para permanecer y que la continua lucha de clases sólo radicalizaba a la clase trabajadora, se inició proceso político y económico de cooptación, o eufemísticamente hablando, de cultivo de un "sindicalismo razonable en los negocios". Con la política del "New Deal", Franklin Roosevelt vio cumplidos sus "deseos constantemente expresados de que el capital y el trabajo descansaran juntos como el león y èl cordero" (Josephson). Los dirigentes de la AFL (Federación Americana del Trabajo) voluntariamente respondieron a este galanteo, y hasta el CIO (Congreso de las Organizaciones Industriales) sucumbió, a pesar de que la clase trabajadora estaba perdiendo sus logros. Estos beneficios fueron desgastados por la nueva directiva en el gobierno reflejados en un Congreso reaccionario: 
Las elecciones para el Congreso de 1942 le dieron posesión al Congreso más conservador que Washington había visto en la última década. Durante el siguiente año el Congreso aprovechó toda oportunidad para repudiar el ánimo reformista de los treintas. . . La autorización en junio del Acta Smith-Connally. ... concedió, en base a un gobierno reacio, nuevos poderes para acabar con los sindicatos y con las disputas de la fuerza de trabajo. El congreso rechazó los límites salariales de \$25,000 dólares de salario neto, liquidó la Administración Nacional de la Juventud, hostigó la Administración de Seguridad Agrícola, y buscó terminar de diversas formas con las erogaciones pacificistas (Blum, 1968, p. 746).

La otra cara de la estrategia en contra de la fuerza de trabajo fue el acelerado ataque en contra de la izquierda y de los elementos partidarios de la militancia independiente. En este sentido contribuyó la guerra a través del auspicio de un clima antidemocrático de persecución política, que ganó fuerza durante el período de la posguerra, con el surgimiento de la "guerra fría" en el exterior, y de su corolario interno, el macartismo.

\section{Macartismo e inmigración}

El macartismo representó la estrategia política e ideológica de la dominación del estado corporativo de los Estados Unidos en la Segunda Guerra Mundial y en la era de la "Guerra Fría". El Acta Smith, autorizada en 1940 (también conocida como "El Acta de Registro de Extranjeros"), aunque en un principio se usó en contra de los americanos de ascendencia japonesa bajo el Decreto Ne 9066 de Roosevelt, fue una propuesta de ley evidentemente política, que pretendía ser utilizada por las agrupaciones de inteligencia y de control político. Durante el período de la posguerra y de la guerra coreana, esta acta se convirtió en el fundamento para una gran "cacería de brujas" en contra de los comunistas y de los dirigentes de los trabajadores. A principios de 1947, tres años antes del surgimiento de la campaña de MeCarthy, el presidente Truman expidió un decreto en el que se creaba el Programa Federal de la Lealtad de los Trabajadores para probar la fidelidad de los empleados del gobierno. Los métodos antidemocráticos de esta "prueba de lealtad" fueron hechos aún más anticonstitucionales, cuando después Truman cambió la regla a la creencia de que "una duda razonable sobre la lealtad de una persona" era suficiente para su destitución.

El $80^{\circ}$ Congreso reflejó esta estrategia de igual forma; al mismo tiempo que aprobó estas políticas de Truman, extendiendo la dominación de los Estados Unidos a través de la doctrina de Truman y del Plan Marshall, e institucionalizando el reclutamiento en el país, el Congreso atacó los derechos de los trabajadores particularmente con la autorización en 1947 de la ley Taft-Hartley, refiriéndose al trabajo como el "Acta de trabajador-esclavo". El $81^{\circ}$ Congreso rechazó los intentos creados 


\section{OPERACION ESPALDA MOJADA Y OPERACION GUATEMALA}

para derogar el Acta Taft-Hartley. Se rehusó a considerar las iniciativas de ley sobre derechos civiles y otras medidas populares. El triunfo de la Revolución China en 1949, y el reto a los Estados Unidos en el monopolio de la bomba atómica por parte de los soviéticos en 1950 , fueron las principales brechas en el control internacional de los Estados Unidos. Por esto, los Estados Unidos incrementó su "guerra fría" tanto interna como externamente.

En 1948, el caso Alger Hiss sirvió como escalón para que Richard Nixon se lanzara al público políticamente, seguido por la campaña antiroja de MeCarthy; desplegada a principios de 1950. Poco después de que por primera vez McCarthy inició su cruzada, Truman aceptó el documento del Consejo de Seguridad Nacional (NSC-68). Este documento, al que Truman llamó su "plan de cinco años para la paz", apresuraba a los Estados Unidos para que se comprometiera:

en un 'atrevido programa masivo de reconstrucción del potencial defensivo en el oeste para rebasar el del mundo soviético',

Los diseñadores del NSC-68. . . recomendaron que Truman. . . preparara a la nación para la defensa, gastando hasta el 20 porciento del producto nacional bruto, en la escala de 50,000 millones anualmente. . . De muchas formas, el documento NSC-68 iba aún más lejos que la doctrina de Truman, ya que significaba que Estados Unidos se convirtiera en una nación militarizada, aceptando la carga, en tiempos de paz, de un aparato militar grande y permanente. La seguridad nacional era ahora definida en términos globales; la represión se expandió a la contienda militar con los soviéticos por el control del mundo. (Divine, 1979, p. 31) (subrayado nuestro).

El primer fruto internacional de esta estrategia fue el hecho de que Estados Unidos se involucrara de inmediato en la guerra de Corea.

Con la escalada internacional en contra del socialismo y la lucha por la hegemonía de los Estados Unidos, el Acta Smith, la ley Taft-Hartley, el Juramento de Lealtad, el Programa Bracero, y otras medidas tomadas durante y después de la guerra, fueron consideradas como armas insufi. cientes en la guerra fría interna. En septiembre de 1950, el Acta de Seguridad Interna de McCarran fue autorizada; ésta demandaba el registro de los comunistas prohibiendo su contratación en la industria de defensa. estableciendo los lugares a los cuales se les negaba el pasaporte, o bien, ordenando la deportación de cualquier inmigrante que hubiera sido miembro del partido comunista. La ley también estipuló las detenciones en caso de "emergencia nacional" de "cualquier persona de la cual hay motivos para creer que pudiera involucrarse en actos de espionaje o sabotaje" ". (Divine, 1979, p. 39). La suspensión de los derechos constitucionales que se habían aplicado a los "extranjeros" japoneses y a los japonesesamericanos durante Ia Segunda Guerra Mundial, ahora se hacía extensiva a todos los ciudadanos norteamericanos y a los extranjeros. Esta acta fue apoyada por los líderes demócratas del Congreso quienes "apresuraban al 
presidente para que aceptara estas medidas a pesar de sus flagrantes violaciones a las libertades individuales (Divine, 1979, p. 39) (subrayado nuestro).

Siete meses después, en abril de 1951, en Washington, D.C. se llevó a cabo la Cuarta Conferencia de Ministros Latinoamericanos, en la cual Estados Unidos pugnó por la cooperación militar y de inteligencia en la "lucha contra el comunismo". Dentro de las medidas adoptadas en la conferencia estaba la recomendación a los gobiernos latinoamericanos para que:

examinaran sus leyes y reglamentos y adoptaran 'aquellas modificaciones. . necesarias para asegurar que las actividades subversivas. . . del comunismo internacional. . pudieran ser prevenidas y castigadas; $y$ que [los gobiernos] tomaran medidas para regular. . . el tránsito. . . de aquellos extranjeros de quien razonablemente se puede suponer que intentan cometer actos subversivos. . .' (subrayado nuestro) (Aguilar Monteverde, 1965, p. 127).

Así, los principios fascistas de las actas Smith, Taft-Hartley, y la de Seguridad Interna de McCarran, que fueron impuestos internamente por los Estados Unidos, también requerían que sus vecinos del "patio trasero" la impusieran en sus países.

Un año depués, en 1952, el Congreso de los Estados Unidos autorizó la Ley de Derecho Público 414, también conocida como el Acta Walter McCarran o la de Inmigración y Nacionalidad. Esta ley, conjuntamente con la Acta de Inmigración de 1917 (y sus reformas), tuvo como objetivo establecer las bases de la inmigración de la posguerra hasta la autorización del Acta de 1965. El Acta de 1952, con la justificación de la codificación de todas las leyes de inmigración y naturalización existentes, representó un arrasador ataque político a los derechos de los inmigrantes. El senador estadounidense Herbert H. Lehman la describió como un acta que "conduce a un punto inhumano y severo la doctrina de que los extranjeros no tienen garantizados sus derechos en este país". (Se agregó subrayado) (Morgan, 1954, p. 4).

Pero al igual que el antisemitismo en la Alemania nazi (para quienes el blanco real era la clase trabajadora alemana y sus dirigentes alemanes comunistas), el miedo a los "extranjeros" era sólo una justificación para atacar a la izquierda estadounidense y a las actividades políticas de la clase trabajadora. En 1953, una lección trágica fue dirigida al país, misma que fue dada a las fuerzas democráticas norteamericanas y extranjeras a través de la ejecución de Ethel y Julius Rosenberg; del mismo modo que las ejecuciones de Sacco y Vanzetti en el período posterior a la Primera Guerra Mundial, fueron usadas simbólicamente en las persecuciones políticas de ese período. La ejecución de los Rosenberg en 1953, fue efectuada durante la administración de Eisenhower. En junio de 1953, a pesar de las súplicas y de las protestas en todo el país y en el mundo entero 
en contra de la ejecución de los Rosenberg, supuestamente por dedicarse al espionaje atómico, con base en el Acta de Espionaje de 1917, Eisenhower se negó a conceder clemencia y la Suprema Corte echó abajo la apelación de Justice Douglas para detener la ejecución.

El presidente Eisenhower y su secretario de Estado John Foster Dulles acordaron fortalecer las acciones de MeCarthy y de sus contemporáneos. El "Reporte de noticias de los Estados Unidos y del Mundo", comentó lo provechoso de la histeria de la guerra fría. La edición periodística del 17 de febrero de 1950, establecía que "'Los miedos a la guerra son fáciles de crear, y son casi seguros productores de dinero para más armamentos' ". En una edición posterior, se establecía que,

Los planificadores del gobierno pensaron que habían encontrado la fórmula mágica para casi eternizar la buena época. Ahora empiezan a preguntarse si después de todo quizá haya algo que no perpetúe el movimiento. . . La guerra fría es el catalizador. La guerra fría es una bomba alimentadora automática, se gira la llave, y el público clama por mayores gastos. . . ... los cuales son demandados por la guerra fría, si son explotados al máximo, son casi ilimitados. (Green, 1976, p, 43).

De hecho, para 1953, bajo el estímulo de la guerra fría, la guerra de Corea, y la armamentización masiva amparada por el NSC-68, casi el 70\% del presupuesto federal fue dedicado a los gastos de la defensa nacional (Divine 1979:56).

Por tanto, la alerta roja continuó con el despido en los gobiernos estatales de los simpatizantes comunistas en el área de educación, requiriendo juicios de lealtad; imponiendo programas de seguridad. Actores, escritores, directores, estaban en la lista negra; y en Washington, el presidente Eisenhower empezó a mostrar los potenciales "riesgos de la seguridad", muy ampliamente definidos (Divine, 1979, p. 61). Estos ataques ocurrieron, como se mencionó anteriormente, en el contexto de las crisis domésticas e internacionales que enfrentaba la administración de Truman. Internacionalmente, el respaldo norteamericano al gobierno de Corea Sur, estaba en peligro de caer. Reporteros extranjeros comentaron que el régimen de Singman Rhee estaba fortaleciendo una creciente oposición pública que podía hacerlo caer en cualquier momento. El 19 de junio, John Foster Dulles, durante un viaje al Pacífico, manifestó el apoyo de los estados al gobierno de Singman Rhee, en la medida en que él continuara desempeñando su papel "dentro de nuestros grandes proyectos". El 21 de junio anunció: "Corea no está sola. Mis conversaciones con el general MacÁrthur serán seguidas de una acción efectiva". Los acontecimientos se dieron muy rápidamente después de eso:

El 25 de junio, en medio de un clima de confusión y desorden, el reportero John Gunther anunció desde Tokio que, de acuerdo con la información recibida por los oficiales norteamericanos comisionados a esa ciudad, Corea del Sur apenas unas horas antes había atacado a Corea del Norte; pero 
en el transcurso de ese mismo día el gobierno de Estados Unidos oficialmente se dirigió al Secretario General de las Naciones Unidas, denunciando la agresión de Corea del Norte; y antes de que el Consejo de Seguridad se pudiera reunir y estudiar los hechos, [Estados Unidos] ordenó el envío inmediato de tropas en apoyo del tambaleante y antidemocrático gobierno de Rhee, sin considerar que esto constituía una violación de los Artículos 27, 39 y 43 del Tratado de San Francisco. Bajo una fuerte presión de los Estados Unidos, el Consejo de Seguridad, por voto mayoritario, declaró agresor a Corea del Norte inciando así una guerra completamente innecesaria, que le costaría cientos de miles de vidas tanto a Corea como a China y a los Estados Unidos. ${ }^{5}$ (subrayado nuestro) (Aguilar Monteverde, 1964, p. 124; 125).

Cinco años después de "la guerra para terminar con todas las guerras", Estados Unidos estaba inmerso en otra guerra importante. Sin embargo, ésta fue hecha sin consultar a la población norteamericana. Truman autorizó al general MacArthur que iniciara el ataque aéreo, y el 30 de junio le ordenó a las tropas terrestres estadounidenses que penetraran en Corea del Sur. En esto, "él actuó basado sólo en su autoridad como Comandante en Jefe", usurpando así el papel del Congreso al declarar la guerra (Divine, 1979, p. 36). Temiendo la reacción del pueblo norteamericano ante esta nueva, injustificable, y unilateral complicación, Truman,

presentó el conflicto al pueblo norteamericano como una violación del Acta Constitutiva de las Naciones Unidas, sosteniendo en una conferencia de prensa que los Estados Unidos no estaba comprometido en una guerra pero, en cambio, era un intento "para suprimir una incursión bandolera en la república de Corea". Cuando un reportero preguntó que si era un llamado certero a la lucha "una acción policial auspiciada por las Naciones Unidas", el presidente contestó, "Sí, eso es exactamente lo que pretende". Durante los tres años siguientes, Estados Unidos mantuvo la ficción de que la guerra en Corea era simplemente una medida policial de las Naciones Unidas. (subrayado nuestro) (Divine, 1979, p. 36).

En el escenario interno, en 1950 y 1951 se presentaron una serie de escándalos que mostraban la corrupción y el crimen del gobierno. Una investigación del senado en 1950, encabezada por un senador políticamente ambicioso, "hizo serios daños políticos al revelar los nexos estrechos entre las maquinarias establecidas en las ciudades, que estaban controladas por los demócratas, y los sindicatos criminales" (Divine, 1979, p. 39). Al mismo tiempo, en diciembre ante una exitosa e inesperada ofensiva de Corea del Norte, Truman decidió presionar para obtener un presupuesto para la defensa nacional de "más o menos $\$ 52 \mathrm{mil}$ millones, cuatro veces el nivel de gastos antes de la guerra coreana", declaraba un estado de "emergencia nacional", y anunciaba los proyectos para aumentar los impuestos y el control de los precios y de los salarios (Divine, 1979, p. 41).

\footnotetext{
${ }^{5}$ Mi traducción del español. En el Reporte Gunther, Aguilar Monteverde cita a I.F. Stone (1952, p. 45).
} 
En 1951, Truman acabó con otra tormenta cuando destituyó al general MacArthur del mando. Después le siguieron más escándalos, al revelarse la corrupción entre las personas apoyadas y nombradas por él mismo. Para culminar, el atolladero en Corea y la creencia de que Estados Unidos estaba involucrado en una guerra de la cual ya se sabía que no podía ganar, Truman se sumó a la propagación del descontento público.

Fue en la atmósfera de estos acontecimientos que los principales periódicos tales como The New York Times, Life, y Time, repentinamente descubrieron la "invasión de los espalda mojada" (Tomasek, 1961, p. 313; García, 1980, pp. 143-144). Hasta finales de 1951, la prensa había ignorado el tema de la inmigración indocumentada. Entonces, de repente:

Aparentemente, de la noche a la mañana el público fue inundado de una gran cantidad de artículos y reportajes especiales sobre los trabajadores indocumentados. La mayoría de los artículos y reportajes manifestaban los efectos negativos que este descontrolado flujo significaba en la economía y en el bienestar social. Lamentaban la gran cantidad de daños que representaba la entrada ilegal, culpando a este movimiento del incremento en las tasas de enfermedades, criminalidad y tráfico de narcóticos, inerementando los costos de beneficiencia y la infiltración de elementos subversivos. (García, 1980, p. 143).

\section{El Acta de Inmigración y Nacionalidad de 1952}

La coordinación de políticas internas y externas que se desarrollaron en este período fue sumamente efectiva en la persecución de los objetivos del capitalismo de estado. En lo referente a la inmigración y a la política exterior, esta coordinación demostró ser crucial, como ya hemos visto, en el papel de deportar a los "extranjeros ilegales" y al camuflar y desviar la atención del público norteamericano y latinoamericano del asunto en el cual estaba directamente involucrado Estados Unidos: el derrocamiento de los gobiernos legítimos de Latinoamérica. El Acta Walter-McCarran de Inmigración y Nacionalidad de 1952, sirvió como un instrumento útil en este asunto, así como lo hizo en el control de la fuerza de trabajo interna e importada.

El Acta Walter-McCarran fue la base para lo que el gobierno y la prensa llamaron "Operación Espalda Mojada" y que mucha gente llamó "Operación Terror", Ilevada a cabo en 1954 (Morgan, 1954). También fue la base de la continua importación de fuerza de trabajo mexicana después de la "Operación Espalda Mojada" y después de que finalizó el programa Bracero en 1964. Esta acta constituyó un elemento importante de la estrategia del gobierno corporativo estadounidense en contra de la izquierda y de los trabajadores internos; mientras avanzaba en sus estrategias económicas y políticas de control externo. 
En casa, las actas McCarran y la Walter McCarran fueron usadas como un ataque en contra de la fuerza de trabajo mexicana y estadounidense. Los ataques a los mexicoamericanos justificados por estas leyes fueron combatidos por varias organizaciones creadas para defender los derechos políticos de los inmigrantes. Una de éstas fue el Comité de Los Angeles para la Protección de los Nacidos en el Extranjero. En un reporte cuidadosamente documentado: Shame of a Nation ("Vergüenza de una Nación"), el Comité examinó los ataques políticos en contra de la comunidad mexicoamericana. Con referencia a las actas McCarran y WalterMcCarran, el estudio sostenía que:

Estas leyes han abolido el decreto de las limitaciones y crearon las bases retroactivas para la deportación, aún cuando la Constitución de los Estados Unidos prohíbe tales leyes ex-postfacto.

Estas leyes por sí solas legalizan muchas de las prácticas usadas ilegalmente en los últimos años por el Departamento de Justicia y su Servicio de Inmigración y Naturalización en contra de los mexicoamericanos (Morgan, 1954, 37-38).

El Comité de Los Angeles para la Protección de los Nacidos en el Exterior, señaló cómo las leyes McCarran y Walter-McCarran fueron aplicadas retroactivamente para perseguir a los mexicoamericanos, que habían estado involucrados con el Partido Comunista o con las Alianzas de los Trabajadores y con los Consejos de los Desempleados durante la Gran Depresión. Entre sus víctimas se encontraban los líderes de la comunidad que habían luchado en contra de la segregación escolar de los niños mexicanos en el Condado de Orange, de los que se encontraban involucrados en la huelga marítima Internacional de Estibadores en 1923, etc. El advirtió que:

Los trabajadores acereros, operadores de máquinas, empacadores, esmeriladores, ferrocarrileros, carpinteros, hojalateros, zapateros, jornaleros, pizcadores y empacadores de fruta, trabajadores agrícolas: la lista de los mexicanos nativos deportados de Los Angeles es una prueba adecuada de que la Ley Walter-MeCarran es dirigida directamente al corazón de la fuerza de trabajo norteamericana. Con esta ley, so pretexto de cazar 'ilegales' y 'subversivos,' los oficiales del Servicio Inmigración fungieron como una fuerza policiaca terrorista en las comunidades mexicanas, como rompehuelgas, como fuerza domadora de los sindicatos en el campo, comercios e industrias. (Morgan, 1954, p. 39).

Más que esto, la Ley de 1952 fue un paso adicional en las agresiones federales a los derechos de los trabajadores inmigrantes. El Comité de Los Angeles señaló que la ley fue autorizada "en el centro de la guerra de Corea y al final de la campaña 'anti-extranjera' que duró cinco años, en el cual el Departamento de Justicia de los Estados Unidos sufrió numerosos retrocesos al buscar la destitución de los derechos constitucionales de los no ciudadanos" (Morgan, 1954, p. 4). 
Con la autorización de la ley de 1952, Ia deportación de inmigrantes indocumentados fue fuertemente acelerada. Entre 1950 y 1951, las deportaciones se incrementaron en un $7 \%$ (a 518,000$)$; en 1952, ascendieron $15 \%$ y en 1953 subieron $40 \%$ (a 876,000$)$. De enero a junio de 1954 (antes de la "Operación Espalda Mojada"), 479,458 mexicanos y mexicoamericanos fueron deportados (American Committee, 1974, pp. 3334). Y no obstante, las deportaciones masivas de la Operación Espalda Mojada en el verano de 1954, no fueron sólo una escalada de este proceso de política interna, sino que respondieron, como se ha tratado de demostrar, a intereses de largo alcance de las políticas externas del gobierno corporativo estadounidense.

\section{INTERPRETACIONES DE LA "OPERACION ESPALDA MOJADA"}

Los estudios acerca de la "Operación Espalda Mojada" han tendido a enfocarse a los acontecimientos inmediatos que giraron en torno a la operación y, excepto de la discusión de la respuesta de México al asunto, los análisis generalmente no han considerado el contexto de los acontecimientos internacionales y de las estrategias políticas estadounidenses para el exterior.

Las explicaciones de la operación, por tanto, se han centrado en el modelo del mal trato de Estados Unidos para los inmigrantes mexicanos y para los mexicoamericanos, en los ataques de McCarthy, en la represión de ese período, y en la recesión de 1953-54. Mientras que los dos primeros son ciertamente parte de la explicación; en relación con la recesión, aun cuando los ataques de la prensa a los extranjeros ilegales sirvieron como chivo expiatorio de los males económicos y sociales de la nación, la "operación" no se llevó a cabo sino hasta mediados de 1954 cuando ya se había iniciado la recuperación. Más aún, en medio de la recesión en enero de 1954, el gobierno de Estados Unidos se comprometió en un reclutamiento masivo ilegal de fuerza de trabajo mexicana en la frontera. De hecho, el gobierno de Estados Unidos cooperó con los agricultores para construir activamente la reserva de fuerza de trabajo de los "espalda mojada", para convertir a los "ilegales" en trabajadores legales contratados temporalmente (braceros), y para convertir a braceros en "ilegales".

Las explicaciones tradicionales no consideran la gran cantidad de discrepancias en la política en torno a la "operación", ni explican, satisfactoriamente, las preguntas surgidas.

Aún cuando se aplica un análisis político-económico a los acontecimientos nacionales e internacionales en torno a la "Operación Espalda Mojada", surge una imagen notoria de la coincidencia de intereses y sucesos entre este acto de racismo masivamente orquestrado, y las estrategias estadounidenses de la guerra fría. La victoria real de la "Operación 
Espalda Mojada" no fue en contra de la amenaza de la "invasión de hordas de miles", extranjeros ilegales destituidos y despojados de sus derechos políticos; sino que ha de encontrarse en la victoria de la "Operación Guatemala", que fue llevada en contra de la amenaza de un gobierno democrático latinoamericano interesado en asegurar el bienestar de su población a través de la expulsión de la verdadera fuerza que ha perpetuado "el desmembramiento" de su economía. 


\section{BIBLIOGRAFIA}

ACUÑA, Rodolfo. Occupied America: A History of Chicanos. New York, Harper \& Row. 1981.

AGUILAR Monteverde, Alonso. El panamericanismo de la Doctrina Monroe a la Doctrina Johnson. México, Cuadernos Americanos. 1965.

AMERICAN Committee for Protection of Foreign Born. Our Badge of Infamy, A Petition to the United Nations on the Treatment of the Mexican Inmigrant (1959) reimpreso en: The Mexican American and the Law. New York, Arno Press. 1974.

BARNET, Richard J. Roots of War; The Men and Institutions Behind U.S. Foreign Policy. Baltimore, Maryland, Penguin Books. 1972.

BENNETT, Lerone Jr. Before the Mayflower: A History of the Negro in America 1619-1964. Revised Edition. Baltimore, Maryland, Penguin Books. 1966.

BLUM, John M. and Bruce Catton, et al. The National Experience. Part Two. A History of the United States Since 1865. New York, Harcout, Brace \& World. 1968.

CITIZEN'S Committee for the Deense of Mexican-American Youth. The Sleepy Lagoon Case. Los Angeles, California, 1942. Reimpreso en: The Mexican American and the Law. New York, Arno Press, 1974.

COCHRAN, Thomas C. American Business in the Twentieth Century. Cambridge, Harvard University Press. 1972.

DIVINE, Robert A. Since 1945; Politics and Diplomacy in Recent American History. New York, Joan Wiley \& Sons. 2a. ed. 1979.

DOBB, Maurice. Capitalism, Yesterday and Today. New York, Monthly Review Press. 1962.

ENDORE, Guy. Justice for Salcido. Los Angeles, California. Civil Rights Congress of Los Angeles, 1948. Reimpreso en: The MexicanAmerican and the Law. New York, Arno Press. 1974.

FREYMOND, Jacques. Western Europe Since the War, A Short Political History. New York, Praeger. 1964.

GALARZA, Ernesto. Merchants of Labor, The Mexican Bracero Story. Santa Barbara, McNally \& Loftin, West. 1978.

GARCIA, Juan Ramón. Operation Wetback: The Mass Deportation of Indocumentated Workers in 1954. Westport, Greenwood Press. 1980.

GERASSI, John. The Great Fear. The Reconquest of Latin America by Latin Americans. New York, The McMillan Company. 1963.

GONZALEZ, Isabel. Step-Children of a Nation: The Status of MexicanAmericans. New York, American Committee for Protection of Foreign Born. 1947. Reimpreso en: The Mexican American and the Law. New York, Arno Press. 1974. 
GONZALEZ Méndez, Rosalinda. "Capital Accumulation and Mexican Inmigration to the United States: A Comparative Historical Study of the Political Economy of International Labor Migrations. "Tesis Doctoral, University of California at Irvine. Michigan, University Microfilms. 1981.

GREBLEN, Leo. Mexican Inmigration to the United States: The Record and Its Implications. Mexican American Study Project, Advanced Report No 2. Los Angeless, UCLA Division Research, Graduate School of Business Administration. 1965.

GREEN, Gil. Wat's Happening to Labor. New York, International Publishers. 1976.

GREENLEAF, Barbara Kaye. America Fever, The Story of American Inmigration. New York, Mentor, New American Library. 1974.

GUZMAN, Ralph C. The Political Socialization of the Mexican American People. New York, Arno Press. 1976.

HOROWITZ, David. Corporations and the Cold War. New York, Monthly Review Press. 1969.

HUDSON, Michael. Super Imperialism; The Economic Strategy of American Empire. New York, Holt, Rinehart \& Winston. 1972.

JOSEPHSON Matthew. The Money Lords; The Great Finance Capitalists 1925-1950. New York and Sacarborough, Ontario, New American Library, A Mentor Book, Times Mirror. 1972.

KINDER, Herman and Werner Hilgemann. The Anchor Atlas of World History. Volume II. From the French Revolution to the American Bicentennial. Traducido por Ernest A. Menze con mapas de Herald y Ruth Bukor. Garden City, New York, Anchor Books Press/Doubleday. 1978.

LENS, Sidney. The Labor Wars, From the Molly Maquires to the Sitdowns. Garden City, New York, Anchor Books, Doubleday. 1974.

MCWILLIAMS, Carey. Brothers Under the Skin. Rev. Ed. Boston, Little, Brown \& Company. 1964.

Factories in the Fields, The Story of Migratory y Farm Labor in California. Santa Barbara, Peregrine Publishers. 1971.

Southern California: An Island on the Land. Santa Barbara, Peregrine Smith. 1979.

MORALES, Armando. Ando sangrando (I Am Bleeding): A Study of Mexican American-Police Conflict. Fair Lawn, New Jersey, R.E. Burdick, Inc., by special arrengement with Perspectiva Publishers, La Puente, California. 1972.

MORGAN, Patricia. Shame of a Nation: A Documented Story of Police State Terror Against Mexican-Americans in the U.S.A. Los Angeles, California, Los Angeles Committee for Protection of Foreign Born, 1954. Reimpreso en: The Mexican American and the Law, New York, Arno Press. 1974. 
THE NATIONAL ADVISORY COMMISSION ON CIVIL DISORDERS. Report of The National Advisory Commission on Civil Disorders. Washington, D.C., U.S. Government Printing Office. 1968.

REAVIS, Dick J. Without Documents. New York, Condor Publishing. 1978.

SAMORA, Julián. Los mojados: The Wetback Story. With The Assistance of Jorge A. Bustamante and Gilbert Cardenas. Notre Dame, University of Notre Dame Press. 1971.

STALEY, Eugene. The Future of Underdeveloped Countries: Political Implications of Economic Development. Revised Edition. Published for the Council on Foreign Relations by New York, Praeger. 1961.

STOESSINGER, John G. The Might of Nations; World Politics in Our Time. New York, Random House. 1961.

U.S. President's Commission on Migratory Labor. Migratory Labor in American Agriculture. Report of the President's Commission on Migratory Labor. Washington, D.C.; U.S. Government Printing Office. 1951.

WISE, David y Thomas B. Ross. The Invisible Government. New York, Random House. 1964. 